\title{
A Case of Churg Strauss Syndrome
}

\author{
Authors \\ Dr Rajput S G ${ }^{1}$, Dr Saoji N R ${ }^{2}$, Dr Bakre $A^{3}$ \\ ${ }^{1}$ Junior Resident, ${ }^{2}$ Associate Professor, ${ }^{3}$ Lecturer \\ ${ }^{1,2,3}$ Department of General Medicine, NKP Salve Institute of Medical Sciences \& Research Centre, \\ Lata Mangeshkar Hospital, Nagpur \\ Corresponding Author \\ Dr Rajput S G \\ Lata Mangeshkar Hospital, Hingna Road, Nagpur PIN- 440019 \\ Email: sachinaps24@gmail.com, Mobile Number-8806210208
}

\begin{abstract}
Eosinophilic granulomatosis with polyangiitis (Churg-Strauss syndrome) is characterized by asthma, peripheral and tissue eosinophilia, extravascular granuloma formation, and vasculitis of multiple organ systems. It is an uncommon disease with an estimated annual incidence of 1-3 per million. Autoimmunity is the postulated etiologic mechanism of the disease. The characteristic laboratory finding is a striking eosinophilia, which reaches levels $>1000$ cells $/ \mu \mathrm{L}$ in $>80 \%$ of patients. Approximately $48 \%$ of patients have circulating ANCA that is usually antimyeloperoxidase. Histologic demonstration of eosinophilic granulomas is pathognomonic. We report a case of a 42 years old male who presented with asthma, sensorimotor polyneuropathy besides constitutional features viz. weight loss \& anorexia.
\end{abstract}

Keywords-Eosinophilic granulomatosis with polyangiitis, Churg-Strauss syndrome, eosinophilia, ANCA, asthma.

\section{Introduction}

Eosinophilic granulomatosis with polyangiitis (EGPA)/ Churg-Strauss syndrome is characterized by asthma, peripheral and tissue eosinophilia, extravascular eosinophilic granuloma formation and necrotizing vasculitis of multiple organ systems. It is arare disease with an estimated annual incidence of 1-3 per million. The mean age of onset is 48 years with a female-to-male ratio of $1.2: 1^{\text {(1) }}$. Autoimmunity is the probable etiology. The disease can involve any organ in the body with lung involvement being predominant \& skin, cardiovascular system, kidney, peripheral nervous system and gastrointestinal tract among others commonly involved ${ }^{(2)}$. Diagnosis is made on the basis of evidence of asthma with associated clinical features consistent with vasculitis\& peripheral blood eosinophilia. Glucocorticoids \& immunosuppressive agents are the drugs central to treatment.

\section{Case Report}

A $45 \mathrm{yrs}$ old male presented with difficulty in walking due to right foot drop associated with tingling \& burning sensation in both lower limbs since 1 month. He also complained of weakness \& clawing in left hand since 1 month. There was 
history of associated pain in abdomen, too.He also gave history of anorexia with associated weight loss (over $10 \mathrm{~kg}$ ) over a period of 2 months. There was no history of fever, cough, rhinitis or sinusitis, vomitting or diarrhea. He was diagnosed with bronchial asthma 1 year ago for which he was on regular treatment. There was no family history of atopy or bronchial asthma. On generalphysical examination he was vitally stable. He had postinflammatory hyperpigmentation secondary to healed vesicular rash over both feet \& hyperpigmented annular plaques in the inguinal region \& over the buttocks. Bilateral ulnar nerves \& common peroneal nerves were thickened \& nontender. Systemic examination revealed few scattered rhonchi bilaterally \& mild diffuse abdominal tenderness without organomegaly. Neurological examination revealed decreased touch, pain \& temperature sensations over all four limbs. There was left claw hand \&right foot drop. All deep tendon reflexes were $(1+) \&$ bilateral plantars were absent. Blood investigations revealed peripheral eosinophilia with absolute eosinophil count of 1650/mm3 (>10\% of total leukocyte count) \& raised p-ANCA levels. Pulmonary function test revealed findings consistent with asthma. Electromyography \& nerve conduction velocity assessment revealed chronic mixed (demyelinating \& axonal) asymmetrical sensorimotor polyneuropathy in both UL \& LL. Biopsy from the sural nerve revealed reactive inflammation around blood vessels with no evidence of eosinophilic granulomas. ECG \& echocardiography did not reveal cardiac involvement. Chest $\mathrm{x}$-ray did not show any pulmonary infiltrates; also contrast enhanced CT scan of the abdomen done to evaluate abdominal pain was normal. On the basis of clinical features i.e. asthma, mononeuritis multiplex \& peripheral eosinophilia with raised p-ANCA levels a diagnosis of Churg-Strauss syndrome was made. Patient was put on oral steroids starting with $60 \mathrm{mg}$ prednisolone once daily tapered by $10 \mathrm{mg}$ per month\& maintained on $10 \mathrm{mg}$ daily after that. He was also given cyclophosphamide pulse therapy - 500mg in normal saline intravenously once a month for 3 months to induce remission. Patient responded to treatment but still requires low dose steroid therapy i.e. $10 \mathrm{mg}$ once daily to maintain remission; peripheral neuropathy is however persistent.

\section{Discussion}

Eosinophilic granulomatosis with polyangiitis/ Churg-Strauss syndrome is an autoimmune disorder characterized by asthma, peripheral and tissue eosinophilia, extravascular eosinophilic granuloma formation and necrotizing vasculitis involving small and medium-sized muscular arteries, capillaries, veins and venules of multiple organ systems. It presents clinically with constitutional symptoms such as fever, malaise, anorexia and weight loss; lung involvement dominates the clinical picture characterised by recurrent asthmatic attacks and the presence of pulmonary infiltrates ${ }^{(2,3)}$. Mononeuritis multiplex is the second most common manifestation and occurs in up to $72 \%$ of patients. Allergic rhinitis and sinusitis develop in up to $61 \%$ of patients. Skin lesions occur in $\sim 51 \%$ of patients and include purpura in addition to cutaneous and subcutaneous nodules. Although only $14 \%$ patients develop clinically recognisable cardiac illness, cardiac involvement is a major cause of mortality. Approximately $48 \%$ of patients have circulating ANCA that is usually antimyeloperoxidase ${ }^{(1)}$.The American college of Rheumatology proposed the following 6 criteria for the classification of Churg Strauss syndrome:- 1) asthma 2) eosinophilia greater than $10 \%$ on differential white blood cell count 3) mononeuropathy (including multiplex) or polyneuropathy 4) non-fixed pulmonary infiltrates on roentgenography 5) paranasal sinus abnormality and 6) biopsy containing a blood vessel with extravascular eosinophils. The presence of 4 or more of these 6 criteria gives a sensitivity of $85 \%$ and a specificity of $99.7 \%$ for diagnosis of EGPA ${ }^{(4)}$ However, eosinophilia is considered to be the main risk factor consistent with EGPA \&asthma may not necessarily be present in every case; pre-existing asthma is not the requisite condition for diagnosis of EGPA $^{(5,6)}$. EGPA patients with anti-MPO ANCA more often suffer from vasculitis symptoms, such as 
glomerulonephritis, mononeuritis multiplex and alveolar hemorrhage whereas ANCA-negative patients more frequently develop heart involvement (7). Diagnosis of EGPA is made on the basis of clinical features \& associated peripheral eosinophilia $>1000 / \mu \mathrm{L}$; extravascular eosinophilic granulomas are pathognomonic but they may not occur simultaneously ${ }^{(1)}$. Immunosuppressive agents are the mainstay of therapy starting with steroids \& adding other agents like cyclophosphamide if there is severe disease or inadequate response to steroids, especially to induce remission. Most patients require low dose steroids lifelong to maintain remission. Recent studies suggest the use of new drugs such as rituximab and mepolizumab (anti-IL5 antibody) in EGPA as alternative therapy ${ }^{(2)}$. The prognosis of untreated patients is poor, with a reported 5-year survival of $25 \%$. With treatment, prognosis is favourable, with one study finding a 78 -month survival rate of $70-90 \%{ }^{(8)}$. Myocardial involvement is the most frequent cause of death and is responsible for $39 \%$ of patient mortality ${ }^{(1)}$.

In conclusion, a high degree of clinical suspicion in a patient of asthma presenting with multisystem involvement must be maintained to diagnose Churgstrauss syndrome as timely intervention with steroid therapy can prevent cardiac involvement, which may turn out to be catastrophic \&delay in diagnosis $\&$ disease progression worsens prognosis ${ }^{(3)}$.

\section{References}

1. Langford CA, Fauci AS. The vasculitis syndromes. Ch. 385 In: Kasper DL, Hauser SL, Jameson JL, editors. Harrison's principles of internal medicine, 19th ed. New York: McGraw Hill, 2016; p.2186- 2187.

2. Greco A, Rizzo MI, De Virgilio A, et al. Churg-Strauss syndrome. Autoimmun Rev. 2015;14(4):341-8

3. Churg J, Strauss L. Allergic granulomatosis, allergic angiitis, and periarteritisnodosa. Am J Pathol. 1951; 27(2): 277-301.

4. Masi AT, Hunder GG, Lie JT, Michel BA, et al.The American College of Rheumatology
1990 criteria for the classification of ChurgStrauss syndrome (allergic granulomatosis and angiitis). Arthritis Rheum. 1990;33 (8):1094-100.

5. Chen KR, Ohata Y, Sakurai M, et al. ChurgStrauss syndrome: report of a case without preexisting asthma. J Dermatol. 1992;19 (1):40-47.

6. ZengM, Liu X, Liu Y. Eosinophilic granulomatosis with polyangiitis presenting with skin rashes, eosinophiliccholecystitis, and retinal vasculitis. Am J Case Rep. 2016; 17: 864-868.

7. Mouthon L, Dunogue B, Guillevin L. Diagnosis and classification of eosinophilicgranulomatosis with polyangiitis (formerly named Churg-Strauss syndrome). J Autoimmun. 2014;48-49:99-103.

8. Samson M, Puéchal X, Devilliers H, et al. Long-term outcomes of 118 patients with eosinophilicgranulomatosis with polyangiitis (Churg-Strauss syndrome) enrolled in two prospective trials. J Autoimmun. 2013;43(6):60-69. 